\title{
Descrição da larva de último instar e pupa de Epacroplon cruciatum (Aurivillius) (Coleoptera, Cerambycidae, Cerambycinae) e notas biológicas
}

\author{
Juares Fuhrmann ${ }^{1,3}$, Márcio M. de Oliveira ${ }^{2,4}$, Daniela de Cassia Bená ${ }^{1,3}$, Sergio Ide ${ }^{1} \&$ Antonio Batista-Filho ${ }^{2,4}$ \\ ${ }^{1}$ Laboratório de Entomologia Geral, Centro de Pesquisa e Desenvolvimento de Sanidade Vegetal, Instituto Biológico, Av. Conselheiro Rodrigues Alves \\ 1252, 04014-900 São Paulo-SP, Brasil.jufuhrmann@gmail.com; danicoleoptera@gmail.com; ide@biologico.sp.gov.br \\ ${ }^{2}$ Laboratório de Controle Biológico, Centro Experimental Central do Instituto Biológico, Instituto Biológico, Rodovia Heitor Penteado km 3, \\ 13092-543 Campinas-SP, Brasil.mmoliveirabiologo@gmail.com; batistaf@biologico.sp.gov.br \\ ${ }^{3}$ Programa de Pós-Graduação em Entomologia e Conservação da Biodiversidade, Faculdade de Ciências Biológicas e Ambientais, Universidade Federal \\ da Grande Dourados, Rodovia Dourados-Itahum km 12, 79804-970 Dourados-MS, Brasil. \\ ${ }^{4}$ Programa de Pós-Graduação em Sanidade, Segurança Alimentar e Ambiental no Agronegócio, Instituto Biológico, Av. Conselheiro Rodrigues Alves \\ 1252, 04014-900 São Paulo-SP, Brasil.
}

\begin{abstract}
Description of last larval instar and pupa of Epacroplon cruciatum (Aurivillius) (Coleoptera, Cerambycidae, Cerambycinae) and biological notes. Last larval instar and pupa of South American Hexoplonini Epacroplon cruciatum (Aurivillius, 1899) are described and illustrated. Biological notes are also presented.

KEYWORDS. Beauveria bassiana; Citrus; Heterospilus; Neotropical; timber beetles.

RESUMO. Descrição da larva de último instar e pupa de Epacroplon cruciatum (Aurivillius) (Coleoptera, Cerambycidae, Cerambycinae) e notas biológicas. Último instar larval e pupa do Hexoplonini sul-americano Epacroplon cruciatum (Aurivillius, 1899) são descritos, ilustrados e disponibilizadas notas biológicas.
\end{abstract}

PALAVRAS-CHAVE. Beauveria bassiana; broca-das-pontas; Citrus; Heterospilus; Neotropical.

Hexoplonini (Coleoptera, Cerambycidae, Cerambycinae) contém 151 espécies distribuídas em 21 gêneros, todos registrados na região Neotropical (Clarke 2007; Galileo \& Martins 2009, 2010a, 2010b; Martins 2006; Martins \& Galileo 2010; Martins et al. 2009). Na tribo são conhecidas larvas e pupas de Gnomidolon varians varians Gounelle, 1909, Tetraibidion aurivillii (Gounelle, 1909) e Tapuruia felisbertoi Lane, 1973, descritas respectivamente, por Meyer (1967), Penteado-Dias (1978) e Casari \& Steffanello (2010).

Epacroplon Martins, 1967 (Hexoplonini) inclui E. armatipes (Martins, 1962), registrada para a Bolívia (Santa Cruz) e E. cruciatum (Aurivillius, 1899), que ocorre no Brasil (Bahia ao Rio Grande do Sul), Paraguai e Argentina (Misiones e Corrientes) (Martins 2006).

Epacroplon cruciatum, conhecida popularmente como broca-das-pontas, tem como hospedeiras espécies de Citrus L. (Rutaceae) e é considerada praga potencial para a cultura de cítricos, ao contrario das "falsas-brocas-das-pontas" que possuem uma importância secundária (Silva 1955). Silva (1955) fez notas sobre a biologia de E. cruciatum (sob o nome Hexoplon ctenostomoides Thomson, 1867) da seguinte forma: postura possivelmente efetuada na extremidade dos ramos da copa de Citrus sp.; a larva, ao se alimentar, avança pelo centro do ramo (região medular) comprimindo serragem e excrementos na parte da galeria que vai sendo abandonada; a galeria, de seção elíptica, segue em direção da raiz; o menor diâmetro da galeria possui $1,5 \mathrm{~mm}$ e o maior, ante- rior à câmara pupal, mede cerca 3,0 mm; a larva abandona a região medular para a parte periférica (região meristemática), onde escava a câmara pupal direcionada ao exterior; o orifício de saída é feito pela imago (Fig. 1). A galeria circular enfraquece o ramo que seca e pode cair. Antes do enfraquecimento ou queda do ramo, a presença da coleobroca é difícil de ser notada, pois a larva não descarta a serragem para o exterior. A duração do ciclo é de cerca de um ano.

Silva (1956) observou Leptostylus ovalis Bates, 1863 (Lamiinae, Acanthocinini) (sob o nome Leptostylus neivai Melzer, 1930) e E. cruciatum broqueando o mesmo ramo de Citrus sinensis (L.) Osbeck cv. Pêra, ambas apresentando o comportamento de reter serragem na galeria. Esse comportamento também foi observado em Hylettus seniculus (Germar, 1824) (Lamiinae, Acanthocinini) durante o broqueamento de Citrus sinensis (L.) Osbeck cv. Pêra Rio (Moreira et al. 2003).

A cultura do Citrus é uma das áreas mais importantes do agronegócio brasileiro, sendo o Brasil o maior produtor mundial de laranja (C. sinensis) e responsável por cerca de $60 \%$ da produção mundial do suco de laranja (Neves et al. 2010). Cerambicídeos broqueadores como Diploschema rotundicolle (Audinet-Serville, 1834) (Cerambycinae, Torneutini) ("broca-dos-ponteiros"), Macropophora accentifer (Olivier, 1795) (Lamiinae, Acanthoderini) ("broca-do-tronco") possuem relevância econômica na citricultura desde os primeiros estudos sobre coleobrocas pragas (Bondar 1913, 1915). Surtos 
populacionais de E. cruciatum registrados em 2000, 2005 e 2006, respectivamente, nos municípios de Mogi Guaçu, Itatinga e Taquarituba, Estado de São Paulo, vem demonstrando sua crescente importância (Machado et al. 2007).

Este trabalho disponibiliza ferramenta para a identificação dos imaturos, amplia os registros sobre a biologia e fornece informações tendo em vista o desenvolvimento de pesquisas na área do controle biológico.

\section{MATERIAL E MÉTODOS}

Coletas. Material coletado em Coronel Macedo (2340'05"S, 49¹2'58”O, $639 \mathrm{~m}$ ), em pomar comercial da empresa Agropecuária Castelfranco Ltda. formado por Citrus sinensis (L.) Osbeck cv. Pêra Rio enxertados em C. x limon Osbeck cv. Cravo ("limoeiro"). O município está localizado na região de Avaré, uma das principais áreas citrícolas do Estado de São Paulo. Foram recolhidos ramos com indício de broqueamento em coletas mensais realizadas de janeiro de 2008 a dezembro de 2009.

O nome das plantas segue em acordo com The Plant List (2010). O nome dos cultivares (cv.) está disponível por sua relevância principalmente nas ciências agrárias.

Morfologia. Imaturos coletados foram fixados em etanol $70 \%$ e examinados em estereomicroscópio e microscópio, ambos com câmara-clara acoplada utilizada na confecção dos desenhos. Medições obtidas através do micrômetro ocular do estereomicroscópio. O estudo morfológico baseou-se em Costa et al. (1988), Duffy (1960), Lawrence (1991a, b).

Notas biológicas. Os ramos foram abertos em laboratório e as larvas retiradas e individualizadas em tubos de vidro (18 cm de comprimento por $1,5 \mathrm{~cm}$ de diâmetro) contendo dieta artificial (Machado \& Berti Filho 1999) tampados com algodão hidrófobo esterilizado (Fig. 22). Os tubos foram mantidos em posição inclinada e no interior de salas climatizadas (temperatura $26^{\circ} \mathrm{C} \pm 2$, umidade relativa de $70 \%$ \pm 10 , fotofase de $12 \mathrm{~h}$ ). As dietas foram trocadas de acordo com o consumo.

Coleções. Material estudado ou depositado: Coleção Entomológica, Departamento de Ecologia e Biologia Evolutiva, Universidade Federal de São Carlos, São Carlos (DEBE); Coleção de Micro-organismos Entomopatogênicos Oldemar Cardim Abreu, Centro Experimental Central, Instituto Biológico, Campinas (IBCB); Coleção Entomológica Adolph Hempel, Centro de Pesquisa e Desenvolvimento de Sanidade Vegetal, Instituto Biológico, São Paulo (IBSP).

\section{RESULTADOS}

Larva (Figs. 2-14). Ortossomática, cilíndrica, levemente achatada dorso-ventralmente no tórax e nos segmentos abdominais I e II (Figs. 2, 3). Comprimento: 17,3-22,5 mm; maior largura: 3,2-3,5 mm (metatórax); largura dos segmentos abdominais III-IX: 2,4-2,5 mm; peso: 0,12-0,18 g. Coloração creme, mandíbulas marrom-escuras, áreas adjacentes às peças bucais e escleritos espiraculares ferruginosas. Late- rais com pelos finos, esparsos e claros, parte dos protorácicos inseridos em pontos escuros.

Cabeça (Figs. 4, 5). Prognata, esclerotizada, fortemente retraída no protórax. Sutura epicranial ausente. Gula triangular. Epicrânio deprimido longitudinalmente. Um estema posterior e contíguo às antenas de cada lado. Sutura epistomal distinta. Parte das inserções musculares visíveis como manchas alongadas na área posterior da cabeça. Forâmen occipital dividido em anterior e posterior. Antenífero membranoso, bem desenvolvido. Antenas (Figs. 6, 7) 3-segmentadas; antenômero I quase totalmente inserido no antenífero; apêndice sensorial (segmento suplementar) interno no ápice do antenômero II; antenômero III com cerda apical. Clípeo reduzido, liso, glabro, trapezoidal. Labro pentagonal, pilosidade abundante. Epifaringe (Fig. 8) com quatro sensórios e dois pelos na região mediana; laterais com dentículos; área posterior com pontos grandes distribuídos assimetricamente entre os lados. Mandíbulas (Figs. 9, 10) simétricas; robustas; glabras; região apical côncava, margem cortante; região basal romba, lisa e plana. Maxilas (Figs. 11, 12) simétricas; cardo com pelo láterodistal, face dorsal membranosa com espinhos diminutos no ápice; estipe com pilosidade longa na face ventral e lateral externa, face dorsal com espinhos diminutos na área médiointerna; mala com 9 cerdas, face dorsal com espinhos diminutos apicais; palpífero com espinhos diminutos na área dorsal; palpos 3-segmentados, palpômero I com dois pelos na lateral externa, II com pelo nas laterais interna e externa, III com pelo ventral. Lábio (Fig. 12) com submento retangular, transversal, glabro e pouco esclerotizado; mento trapezoidal com pilosidade densa nas laterais; pré-mento quadrangular, mais ou menos arredondado e com pilosidade densa na metade anterior; palpos 2-segmentados e subcontíguos, palpômeros glabros; lígula densamente pilosa, dividida em lobo médio pequeno e dois lobos maiores laterais à hipofaringe. Hipofaringe (Fig. 13) dividida em área anterior densamente pilosa e posterior abruptamente elevada e menos pilosa; região após a elevação plana, lisa, glabra e mais esclerotizada; duas regiões esclerotizadas margeiam a elevação hipofaringeal.

Tórax. (Figs. 2, 3) Protórax mais longo que o meso- e metatórax combinados e com pilosidade mais densa. Noto com três pares de regiões esclerotizadas: par I transversal e anterior; par II circular, pequeno e mediano; par III transversal, posterior e carenado longitudinalmente. Pleura com pequena área circular esclerotizada. Esterno largo, pilosidade abundante. Basisterno glabro, com par de áreas circulares esclerotizadas laterais. Esternelo glabro, estreito, transverso.

Mesotórax estreito; dorso com duas depressões rasas no contato com o pronoto; espiráculos pleuroventrais, elípticos, maiores que os abdominais.

Metatórax pouco mais longo que o segmento anterior, dorso e ventre enrugados.

Pernas (Fig. 14) reduzidas, largamente separadas e 3-segmentadas; segmentos I e II globulares, com par de cerdas; tarsúngulo alongado e rugoso.

Abdômen (Figs. 2, 3) com dez segmentos bem definidos. Segmentos I-VI com ampolas ambulatórias ventrais e dorsais; 

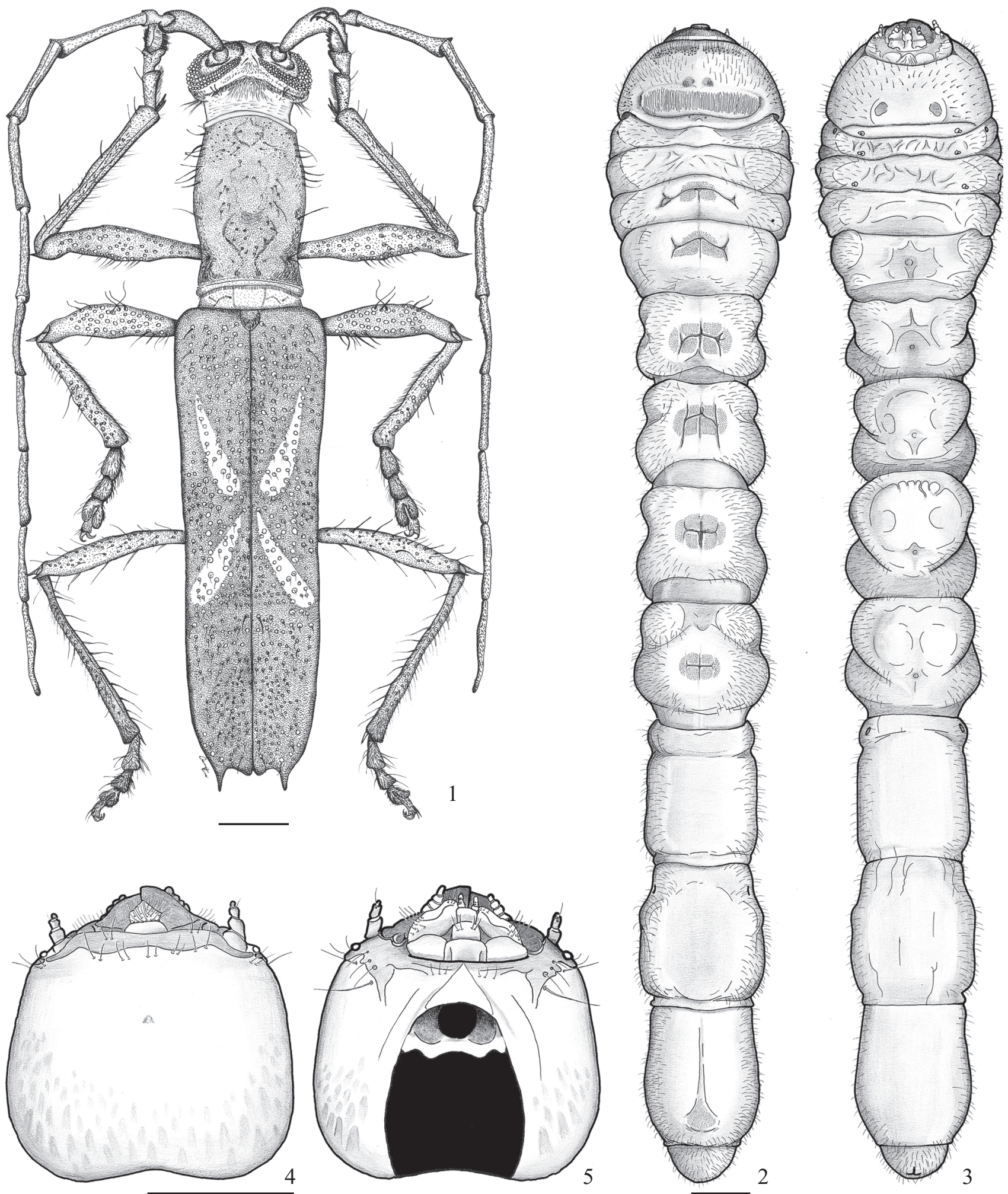

Figs. 1-5. Epacroplon cruciatum (Aurivillius, 1899) (Coleoptera, Cerambycidae, Cerambycinae, Hexoplonini). Imago: 1, habitus (dorsal). Larva, último instar: 2, 3, habitus (dorsal, ventral); 4, 5, cabeça (dorsal, ventral). Escalas $=1,0 \mathrm{~mm}$

dorsais com região média áspera dividida por sulcos longitudinal médio, transversal e dois longitudinais laterais; ventrais 2-lobadas, sem região áspera, com sulcos semelhantes às dorsais, mas pouco distintas. Segmento I encurtado, tão largo quanto o tórax; II mais longo que o I, laterais convergindo posteriormente; III-VI com laterais sinuosas; VII-IX 

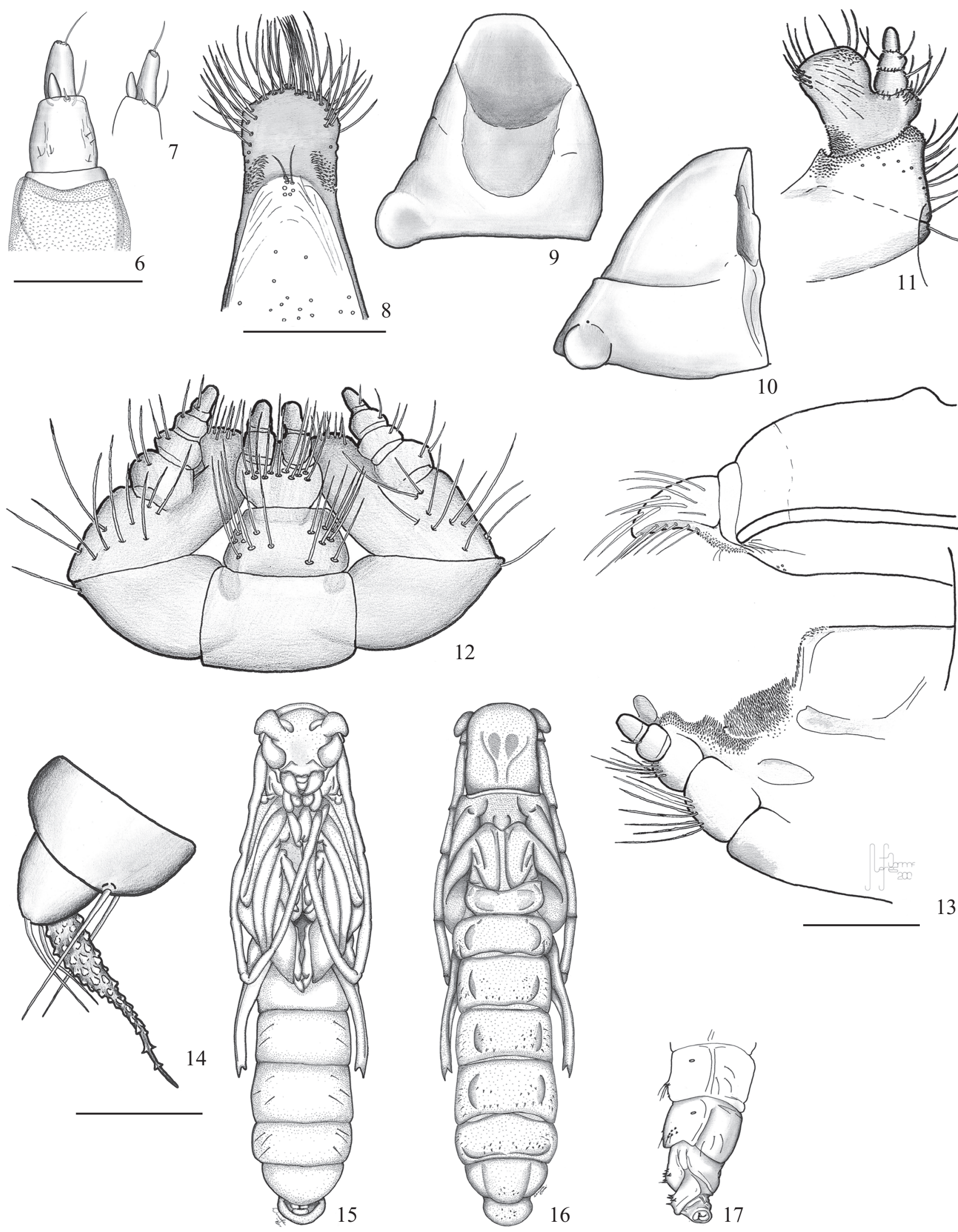

Figs. 6-17. Epacroplon cruciatum (Aurivillius, 1899) (Coleoptera, Cerambycidae, Cerambycinae, Hexoplonini). Larva, último instar: 6, 7, antena (dorsal, lateral interna); 8, epifaringe; 9, 10, mandíbula direita (face interna, dorsal); 11, maxila direita (dorsal); 12, maxilas e lábio (ventral); 13, labro, epifaringe, maxila, lábio e hipofaringe (lateral); 14, perna anterior (lateral). Pupa: 15, 16, habitus (ventral, dorsal); 17, ápice abdominal (lateral). Escalas: 6, $14=0,1$ $\mathrm{mm}, 7-13=0,25 \mathrm{~mm} ; 15-17=2,0 \mathrm{~mm}$. 
alongados, cilíndricos, laterais mais ou menos paralelas; IX com área mesolongitudinal dorsal pouco esclerotizada e rugosa, terminado em tubérculo, urogonfos ausentes; X curto, hemisférico, abertura anal em forma de "T". Espiráculos circulares pleurais nos segmentos I-VIII.

Pupa (Figs. 15-18). Adéctica e exarata. Comprimento: 13,8-15,2 mm; maior largura: 3,5-3,7 mm (nas pterotecas posteriores), largura variando pouco no resto do tronco; peso 0,05-0,07 g. Coloração creme.

Cabeça opistognata, dorsalmente escondida pelo pronoto e ventralmente escondendo o prosterno. Clípeo e labro pequenos e visíveis entre e sobre as mandíbulas. Mandíbulas proeminentes. Escapo cilíndrico, largo, arqueado para trás; pedicelo curto; flagelo filiforme, estendendo-se dorsalmente até a junção mesofêmur-tíbia, estendendo-se ventralmente sobre as pterotecas alares e dobrado próximo ao segmento abdominal III. Palpos maxilares alongados, estendendo-se sobre a junção protíbia-tarso. Palpos labiais globulares.

Tórax. Pronoto alongado; pilosidade fina na borda anterior; par de áreas escurecidas na área central. Escutelo mais ou menos distinto, pequeno, projetado fracamente para trás. Metaescuto dividido por sulco médio, pteroteca posterior encobrindo a junção metatíbia-tarso. Pernas dobradas e repousadas pelo contato da face interna do fêmur com a mesma face da tíbia, ficando os tarsos opostos em contato pela face ventral ou sobrepostos; par de espinhos apicais do metafêmur distinto.

Abdômen (Figs. 15-17) cilíndrico, estreitado no ápice; laterais com pilosidade longa e escassa; tergitos III-VIII com dentículos setígeros mais esclerotizados; urogonfos ausentes. Esternito I extremamente reduzido; II estreitado; segmentos III-VI cilíndricos; VII com forma mais ou menos opercular; expondo o tergito VIII. Esternito VIII membranoso, reduzido, parcialmente menos escondido; segmento IX membranoso; segmento $\mathrm{X}$ reduzido e representado por três pequenos lobos periféricos à abertura anal (Fig. 17) em forma de "T".

Material examinado. BRASIL, São PAUlo: Coronel Macedo, broqueando Citrus sinensis, VII.2008, M. M. Oliveira leg., 2 larvas, 2 pupas (IBSP); IX.2008, M. M. Oliveira leg., 1 larva (IBSP); 27.III.2009, M. M. Oliveira leg., 1 larva (IBSP); 13.VI.2009, M. M. Oliveira leg., 1 larva (IBSP).

Notas Biológicas. Além do indicado por Silva (1955), foi observado que ramos broqueados por E. cruciatum apresentavam murcha seguida por seca de folhas (Fig. 19), extravasamento de seiva (Fig. 20) e perda de frutos. O broqueamento ocorreu em ramos de, no máximo, $5 \mathrm{~cm}$ de diâmetro.

Larvas ocorreram de janeiro a agosto em 2008 e janeiro a julho em 2009, pupas de junho a agosto de 2008 e 2009 e imagos de setembro a dezembro de 2008 e setembro a novembro de 2009. Até duas larvas foram observadas em um mesmo ramo (Fig. 21), porém, em galerias distintas. Foram encontradas larvas infectadas (Fig. 22) pelo fungo Beauveria bassiana (Bals.-Criv.) Vuill. (Hypocreales, Cordycipitaceae) e o fungo isolado foi depositado na IBCB sob as referências
IBCB 614, IBCB 615, IBCB 633 e IBCB 634. Também foram observados parasitoides larvais identificados como Heterospilus sp. (Hymenoptera, Braconidae, Doryctinae), os espécimes estão depositados na DEBE. A criação em laboratório com o uso de dieta artificial mostrou-se viável (Fig. 23), embora não se tenham obtido posturas.

\section{DISCUSSÃO}

A larva de Epacroplon cruciatum assemelha-se às de Gnomidolon varians, Tapuruia felisbertoi e Tetraibidion aurivillii por apresentarem par de estemas; pernas com três segmentos, segmentos I e II globulares, cada um com par de pelos látero-distais; segmentos abdominais I-VI com ampolas ambulatórias ventrais e dorsais; tergito IX com pequena área média esclerotizada. Meyer (1967) não especificou o posicionamento das ampolas nem a presença no tergito IX de pequena área média esclerotizada em G. varians. Epacroplon cruciatum apresenta o labro com a borda anterior obtusa, nas demais espécies é arredondada. A epifaringe em E. cruciatum e T. felisbertoi apresenta um par de pelos longos médios, T. aurivillii sem pelos longos médios. As áreas laterais da epifaringe de E. cruciatum tem dentículos que não avançam posteriormente após a altura dos sensórios médios, os dentículos em T. aurivillii e T. felisbertoi avançam posteriormente após a altura dos sensórios médios. Meyer (1967) não descreveu a epifaringe de G. varians. Em G. varians e T. felisbertoi a mala possui dez pelos apicais, ocorrem oito em T. aurivillii e nove em E. cruciatum. A antena de $G$. varians e T. auriviillii apresenta apêndice sensorial tão ou quase tão longo quanto o antenômero III e ápice do antenômero III com dois pelos longos; em T. felisbertoi e $E$. cruciatum o apêndice sensorial é mais curto que a metade do antenômero III e o ápice do antenômero possui um pelo longo e dois curtos, e um pelo longo, respectivamente. O pronoto de E. cruciatum, G. varians e T. aurivillii apresenta faixa posterior de estrias longitudinais, estrias ausentes em $T$. felisbertoi. Tarsúngulo de E. cruciatum, G. varians e $T$. aurivillii estreito, alongado, rugoso e mais ou menos reto, $T$. felisbertoi com tarsúngulo semelhante, mas sinuoso.

A pupa de E. cruciatum, G. varians e T. aurivillii e alongada, mais ou menos cilíndricas; pronoto inerme, mais longo que largo e de lados paralelos; dorso abdominal com espinhos curtos escassos. A pupa de E. cruciatum pode ser diferenciada pelo pronoto com suas áreas dorsais mais escuras. Em $T$. felisbertoi a pupa é mais oblonga; com pronoto tão longo quanto largo, ângulos anteriores proeminentes e dois tubérculos dorsais; dorso abdominal com espinhos curtos semelhantes aos das outras espécies, mas bem mais densos. A metatíbia das pupas de E. cruciatum, G. varians, T. aurivillii e $T$. felisbertoi apresentam, 2, 2, 1, 0 espinhos apicais, respectivamente.

A câmara pupal de G. varians é alongada, longitudinal ao ramo e pode ficar próxima a casca ou no interior do ramo; a de T. aurivillii é alongada, longitudinal e no centro do ramo; a de $T$. felisbertoi é oval, periférica ao ramo e observada ex- 

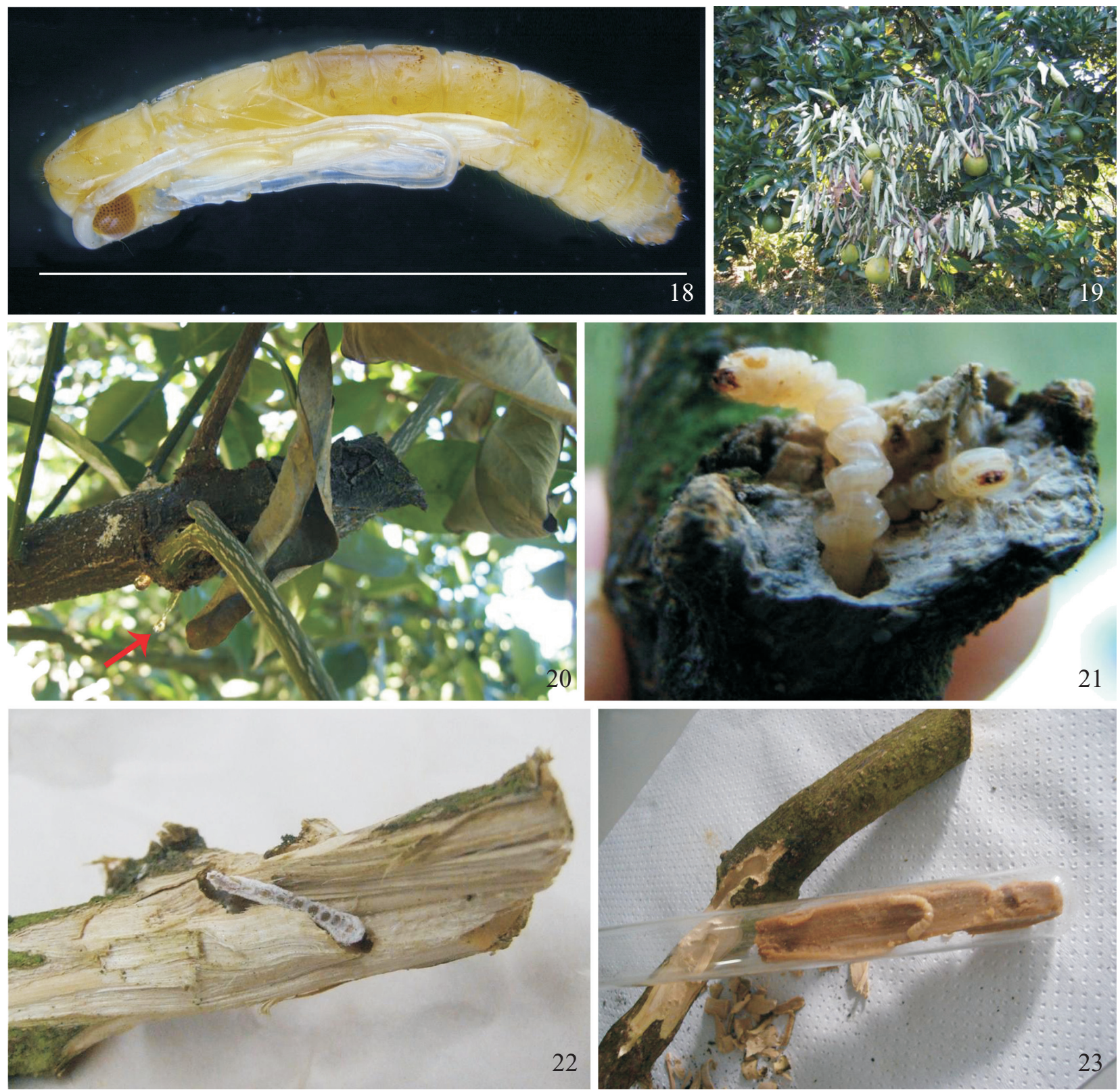

Figs. 18-23. Epacroplon cruciatum (Aurivillius, 1899) (Coleoptera, Cerambycidae, Cerambycinae, Hexoplonini). Pupa: 18, habitus (lateral), Escala =13 mm. Sintomas de infestação em Citrus sinensis (L.) Osbeck cv. Pêra Rio (Rutaceae): 19, seca de ramo; 20, extravasamento da seiva ("mela"). Danos: 21, larvas em galerias; 22, larva em galeria morta por Beauveria bassiana (Bals.-Criv.) Vuill. (Hypocreales, Cordycipitaceae); 23, galerias e larva em dieta artificial.

ternamente por uma leve convexidade; e a de E. cruciatum é alongada, periférica ao ramo e levemente inclinada ao eixo do ramo.

\section{AGRADECIMENTOS}

Ao Dr. Ubirajara R. Martins (Museu de Zoologia, Universidade de São Paulo, São Paulo) pelas críticas e conselhos, à Dra. Angélica Maria Penteado Martins Dias (Universidade Federal de São Carlos) pela identificação do parasitoide, à Coordenação de Aperfeiçoamento de Pessoal de Nível Superior pelas bolsas de mestrado ao Juares Fuhrmann e Daniela de C. Bená e à Secretaria da Educação do Estado de São Paulo (Projeto Bolsa Mestrado) pela bolsa ao Marcio M. Oliveira.

\section{REFERÊNCIAS}

Bondar, G. 1913. Brocas das laranjeiras e outras aurantiáceas. Serviço de Informações e Divulgação, Boletim do Ministério da Agricultura, Indústria e Comércio 2: 81-93.

Bondar, G. 1915. Insectos daninhos a agricultura. III. Pragas das laranjeiras e outras aurantiáceas. São Paulo, Duprai \& Cia., 47 p.

Casari, S. A. \& E. A. W. Steffanello. 2010. Immatures of Tapuruia felisbertoi Lane, 1973 (Cerambycidae, Cerambycinae, Hexoplonini). Papéis Avulsos de Zoologia 50: 425-434. 
Clarke, R. O. S. 2007. New and newly illustrated Gnomidolon Thomson (Coleoptera, Cerambycidae, Hexoplonini) from Bolivia. Revista Brasileira de Entomologia 51: 257-262.

Costa, C.; S. A. Vanin \& S. A. Casari-Chen. 1988. Larvas de Coleoptera do Brasil. São Paulo, Museu de Zoologia, Universidade de São Paulo, v+282 p.

Duffy, E. A. J. 1960. A monograph of the immature stages of Neotropical timber beetles (Cerambycidae). London, British Museum (Natural History), $327 \mathrm{p}$

Galileo, M. H. M. \& U. R. Martins. 2009. Cinco novas espécies de Cerambycidae (Insecta: Coleoptera) da região Neotropical e novos registros. Zoologia 26: 161-165.

Galileo, M. H. M. \& U. R. Martins. 2010a. Novos táxons de Cerambycinae (Coleoptera, Cerambycidae) da América do Sul. Papéis Avulsos de Zoologia 50: 385-390.

Galileo, M. H. M. \& U. R. Martins. 2010b. New species of Cerambycinae (Coleoptera, Cerambycidae) from South America. Insecta Mundi 0115: $1-9$.

Lawrence, J. F. 1991a. Larval morphology, p. 146-177. In: J. F. Lawrence (coord.). Order Coleoptera, chapter 34, p. 144-658. In: F. W. Stehr (ed.). Immature insects, v. 2. Dubuque, Kendall/Hunt Publishing Company, xvi+975 p.

Lawrence, J. F. 1991b. Cerambycidae (Chrysomeloidea) (including Disteniidae, Hypocephalidae, Oxypletidae, Parandridae, Spondylidae, Vesperiidae): long-horned beetles, round-headed wood-borers, p. 556561. In: J. F. Lawrence (coord.). Order Coleoptera, chapter 34, p. 144658. In: F. W. Stehr (ed.). Immature insects, v. 2. Dubuque, Kendall/ Hunt Publishing Company, xvi+975 p.

Machado, L. A. \& E. Berti Filho. 1999. Criação artificial da broca-doscitros Diploschema rotundicolle (Serville, 1834) (Col.: Cerambycidae). O Biológico 61: 5-11.
Machado, L. A.; M. M. Oliveira \& V. B. Silva. 2007. Nova braça em Citrus. Cultivar Hortaliças e Frutas 45: 27-29.

Martins, U. R. 2006. Cerambycidae sul-americanos (Coleoptera), v. 8. Curitiba, Sociedade Brasileira de Entomologia, 230 p.

Martins, U. R. \& M. H. M. Galileo. 2010. Cerambycidae (Coleoptera) da Serra Bonita, Camacan, Bahia, Brasil. Papéis Avulsos de Zoologia 50: $345-443$.

Martins, U. R.; M. H. M. Galileo \& F. Limeira-de-Oliveira. 2009. Cerambycidae (Coleoptera) do estado do Maranhão, Brasil. Papéis Avulsos de Zoologia 49: 229-247.

Meyer, F. R. 1967. Larva, pupa and life history of three species of Ibidionini (Coleoptera, Cerambycinae). Papéis Avulsos de Zoologia 20: $147-$ 158

Moreira, M. A. B.; J. O. L. de Oliveira Júnior \& M. A. Monné. 2003. Ocorrência de Hylettus seniculus (Germar, 1824) (Coleoptera: Cerambycidae), em pomares cítricos de Roraima, Brasil, e alternativas de controle. Acta Amazonica 33: 607-612.

Neves, M. F.; V. G. Trombin; P. Milan; F. F. Lopes; F. C. Pereira \& R. B. Kalaki. 2010. O Retrato da Citricultura Brasileira. Ribeirão Preto, Markestrat, $137 \mathrm{p}$.

Penteado-Dias, A. M. 1978. Biologia e ontogenia de alguns Ibidionini (Coleoptera, Cerambycidae, Cerambycinae). Revista Brasileira de Entomologia 22: 35-48.

Silva, A. G. de A. e. 1955. Seis novas brocas da laranjeira. I. Broca-daspontas. Boletim Fitossanitário 6: 35-44.

Silva, A. G. de A. e. 1956. Seis novas brocas da laranjeira. II. Falsas Brocas das Pontas. Boletim Fitossanitário 6: 119-125.

The Plant List. 2010. The Plant List. Version 1. Disponível em: <http:// www.theplantlist.org $>$. (acessado em 30 de setembro de 2011). 